\title{
A Systematic Review of Enteral Feeding By Nasogastric Tube in Young People With Eating Disorders
}

Kristen Hindley ( $\square$ kristen.hindley@sky.com)

University of Manchester School of Medicine: The University of Manchester Faculty of Biology Medicine and Health https://orcid.org/0000-0002-0001-5056

Clare Fenton

Leeds and York Partnership NHS Foundation Trust

Jennifer Mclntosh

Leeds and York Partnership NHS Foundation Trust

\section{Research Article}

Keywords: nasogastric, enteral feeding, restrictive, eating disorders, young people, adolescents, inpatient, anorexia nervosa

Posted Date: July 13th, 2021

DOI: https://doi.org/10.21203/rs.3.rs-121006/v3

License: () (i) This work is licensed under a Creative Commons Attribution 4.0 International License. Read Full License

Version of Record: A version of this preprint was published at Journal of Eating Disorders on July 22nd, 2021. See the published version at https://doi.org/10.1186/s40337-021-00445-1. 


\section{Abstract}

Background

Adolescents with severe restrictive eating disorders often require enteral feeding to provide lifesaving treatment. Nasogastric feeding (NG) is a method of enteral nutrition often used in inpatient settings to treat medical instability, to supplement poor oral intake or to increase nutritional intake. This systematic review sets out to describe current practice of NG in young people with eating disorders.

Methods

A systematic review following PRISMA guidelines was conducted by searching AMED, EMBASE and MEDLINE databases from 2000-2020. Inclusion terms were: enteral feeding by nasogastric tube, under 18 years, eating disorders, and primary research. Exclusion terms: psychiatric disorders other than eating disorders; non-primary research; no outcomes specific to NG feeding and participants over 18 years. Titles and abstracts were screened by all authors before reviewing full length articles. Quality assessment, including risk of bias, was conducted by all authors.

Results

29 studies met the full criteria. $86 \%$ of studies were deemed high or medium risk of bias due to the type of study: $34.4 \%$ retrospective cohort and $10.3 \%$ RCT; $17.2 \%$ were qualitative. Studies identified 1) a wide range of refeeding regimes depending on country, settings, and the reason for initiation; 2 ) standard practice is to introduce Nasogastric feeds (NG) if medically unstable or oral intake alone is inadequate; 3 ) NG may enable greater initial weight gain due to increased caloric intake; 4) there are 3 main types of feeding regime: continuous, nocturnal and bolus; 5) complications included nasal irritation, epistaxis, electrolyte disturbance, distress and tube removal; 6) where NG is routinely implemented to increase total calorie intake, length of stay in hospital may be reduced; however where NG is implemented in correlation to severity of symptoms, it may be increased; 7) both medical and psychiatric wards most commonly report using NG in addition to oral intake.

Conclusions

NG feeding is a safe and efficacious method of increasing total calorie intake by either supplementing oral intake or continuously. There are currently no direct comparisons between continuous, nocturnal or bolus regimes, which may be used to direct future treatment for YP with ED.

\section{Plain English Summary}

Young people with eating disorders often restrict food intake to a degree which is detrimental to their physical health. Depending on the severity of psychiatric and medical symptoms, patients may be admitted to a mental health or medical ward. In the circumstances that their BMI is detrimentally low, a nasogastric (NG) tube may be placed from nose to stomach to pass nutrition. This systematic review sets out to review the current reported evidence of NG in young people. Results have shown that NG feeding is used commonly in the hospital setting to treat medical instability as a result of severe malnourishment, and in the specialist eating disorders (ED) unit due to failure to meet oral intake. NG feeding may be administered through different methods such as continuously, multiple single meals (bolus), or overnight to supplement day-time oral intake. Routine NG feeding may allow greater initial caloric intake, which does not increase risk of medical complications, and may actually increase initial weight gain thus reducing time in hospital. Differences may be due to variable expertise of staff. Side effects are minimal but may include nasal bleeding or irritation, and imbalances in blood electrolytes which can be reduced by providing supplementation.

\section{Background}

There are currently over 700,000 individuals in the UK with an eating disorder (ED) ${ }^{1}$. EDs usually manifest prior to adulthood, with an average age of onset of approximately 15 years, although this is decreasing; with new research from NICE demonstrating that incidence in children aged 12 and under had increased between 2005-2015 in the UK. ${ }^{2,3}$ Patients with restrictive eating disorders, including anorexia nervosa (AN), bulimia nervosa (BN) and eating disorder not otherwise specified (EDNOS), are predominantly female (91\%) and Caucasian (92\%), with incidence being approximately 0.014 for females. ${ }^{3}$ Compared to other mental illnesses, EDs have a high mortality rate with young people (YP) with anorexia nervosa (AN) on average 6-10 times more likely to die than the general population. ${ }^{4,5}$ Death is often caused by cardiac abnormalities associated with extremely low bodyweight. ${ }^{6}$ For this reason, acute medical intervention is often warranted in order to reduce mortality. Nasogastric (NG) feeding use in YP with ED may be used as a lifesaving treatment when patients are physically unwell. ${ }^{7,8}$ However, refeeding is also a critical component to recovery and NG feeding will often be utilised if a young person has been unable to manage oral intake in order to prevent signs of physical unwellness. 9,10

NG feeding involves a fine bore tube passed via the nasal passage into the stomach in order to administer nutrition. There is a low risk of complications associated with NG feeding if staff receive adequate training and protocols are enforced to ensure that the tube has been passed correctly. ${ }^{11}$ Different methods of NG may be utilised safely, with NG feeds often given as large bolus, continuously through a pump or overnight in order to supplement daytime oral intake. ${ }^{12,13}$ Recent guidance from the British Dietetic Association ${ }^{14}$ for NG feeding under restraint advised $1-2$ bolus feeds per day even in those with high risk of refeeding syndrome (RS); it also concluded further research into this area was required. The National Institute for Clinical Excellence has produced guidance for providing nutrition recommending a graded approach. ${ }^{15}$ Neither of these guidelines are specific for children and adolescents.

Most EDs will be treated in an outpatient setting with hospitalisation generally reserved for those with severe malnutrition resulting in physical symptoms such as bradycardia, hypotension or dehydration as set out in the MARSIPAN guidance. ${ }^{16}$ Research on NG feeding in YP has tended to focus on the acute refeeding

Page 2/16 
phase in paediatric or psychiatric wards to reduce the risk of RS. ${ }^{17} \mathrm{RS}$ can manifest as hypophosphatemia (HP), hypomagnesemia, hypokalemia. and other electrolyte imbalances that result in cardiac arrhythmias, seizures and in some cases sudden death. ${ }^{18}$ During the acute refeeding phase the need for weight restoration must be balanced against the risk of developing RS. Most patients (96\%) however present less severely with serum hypophosphataemia and no clinical signs. ${ }^{19}$ Although there is a significant body of research into this, the role of NG feeding remains ill-defined. ${ }^{17}$

Moreover, for clinicians, there is currently conflicting guidance on how to manage NG feeding in YP with ED, in particular how and when to transition between oral and NG feeding. ${ }^{20,21}$ This has resulted in a variety of NG feeding practices across different settings, with many medical wards tending to provide continuous NG feeds and cease oral intake in order to medically stabilise the patient, ${ }^{20,22-26,}$, in contrast mental health wards or specialized eating disorder programs housed on medical wards may be more likely to use syringe bolus feeds to provide food when meals are refused, encouraging oral intake and aiding normalisation of eating. ${ }^{9,18,27-31}$ In a recent systematic review ${ }^{32}$ 9/10 studies in hospitalised ED patients are given continuous or overnight supplemental NG feeding.

Previous reviews ${ }^{32,33}$ have examined use of NG feeding in ED, including the safety and efficacy of NG feeding as well as short-term and long-term outcomes. However, this will be the first systematic review on the use of NG feeding specifically in YP with ED. Due to the anticipated paucity of studies in this area any research where a meaningful conclusion or result can be drawn regarding NG use in YP with ED will be included. This review aims to assess strategies for the use, tolerance and effectiveness of NG feeding in YP with restrictive ED.

\section{Methods}

A comprehensive database search of AMED, EMBASE, APA Psychinfo and MEDLINE was performed with no language restriction from January 2000 to July 2020. Search strategies combined keywords with controlled vocabulary terms (MeSH, Thesaurus); both quantitative and qualitative research were included. The search criteria was peer reviewed by a researcher from the University of York's Child and Adolescent Mental Health Intervention Centre. References were exported and duplicates were removed using the title and abstract.

\subsection{Screening For Eligible Studies}

The full search is available in Appendix 1. The inclusion criteria were: NG feeding, participants under 18 years, eating disorders, published since 2000 and primary research. The outcomes of interest were: Opinions of YP and staff using NG, amount of YP requiring NG, any interventions that impacted on NG feeding, complications of NG feeding, interventions to mitigate the complications, the setting (medical ward, psychiatric ward or outpatient), the NG method and whether this changed when restraint was required. The exclusion criteria included: No ability to discern results specific to NG feeding, mental disorders other than eating disorders being the focus, where the majority of participants are over 18 years or it is impossible to separate results for adults from YP, reviews or other non-primary research and research published before 2000.

Studies published in languages other than English were translated prior to being reviewed. The PRISMA flowchart was used (Fig. 1). Abstracts identified from the initial search were screened in a secondary review process, and full text papers were obtained of those meeting the inclusion criteria or where there was uncertainty. One article published prior to 2000 was included in the full text review due to it requiring translation prior to assessing it against the criteria. Key studies were manually reviewed for additional research, but none were identified that were not already included, 1 eligible study was identified through peer review. There was no disagreement between $\mathrm{CF}$ and $\mathrm{KH}$ who assessed which studies were included.

Figure 1 displaying PRISMA flowchart of methodology utilised to search databases for this systematic review of enteral feeding in young people with restrictive eating disorders.

\subsection{Assessing Study Quality}

There is no validated method to assess the retrospective and qualitative nature of studies included therefore we could not conduct a formal quality assessment or statistical method to evaluate the results. The risk of bias was estimated into high, medium or low using an adapted version of the Agency for Healthcare Research and Quality risk of bias tool as described in Myers ${ }^{34}$ which included an assessment of the bias in the selection of participants, sample size, tools used to assess change and whether the study involved blinding. The studies were analysed for risk of bias independently by CF, KH and JM. The risk of bias was deemed to be medium or high (see Appendix 2) for the majority of the studies included due to the nature of their design, being case series or retrospective cohort studies. Table 1 includes a summary of included studies. Results interpreted from studies with a high risk of bias were removed accordingly, leaving only high quality results and conclusions. 
Table 1

Summary of Eligible Studies

\begin{tabular}{|c|c|c|c|c|c|c|c|c|c|}
\hline $\begin{array}{l}\text { Study } \\
\text { Design }\end{array}$ & Country Set & $\begin{array}{l}\text { Time } \\
\text { Frame / } \\
\text { Follow up } \\
\text { years } \\
\text { (months) }\end{array}$ & $\begin{array}{l}\mathrm{N} \text { total } \\
\text { (Female) }\end{array}$ & $\begin{array}{l}\text { Age } \\
\text { Range } \\
\text { (years) }\end{array}$ & Setting & Aims & $\begin{array}{l}\text { NG Primary/ } \\
\text { Secondary } \\
\text { Outcome? } \\
\text { (Reason for } \\
\text { Implementing } \\
\text { NG) }\end{array}$ & $\begin{array}{l}\text { Main } \\
\text { Outcomes }\end{array}$ & Risk of Bias \\
\hline $\begin{array}{l}\text { Whitelaw et } \\
\text { al., } 2010^{9}\end{array}$ & $\begin{array}{l}\text { Cohort Study } \\
\text { (retrospective) }\end{array}$ & Australia & TF 1 & $\begin{array}{l}29 \\
\text { (not } \\
\text { stated) }\end{array}$ & $12-18$ & $\begin{array}{l}\text { Adolescent } \\
\text { Medical } \\
\text { Ward }\end{array}$ & $\begin{array}{l}\text { Assess whether } \\
\text { more aggressive } \\
\text { refeeding leaves } \\
\text { patients at } \\
\text { greater risk of } \\
\text { HP }\end{array}$ & $\begin{array}{l}\text { Secondary } \\
\text { (Inadequate } \\
\text { oral intake) }\end{array}$ & $\begin{array}{l}\text { HP associated with lov } \\
\% \text { IBW and lower numb } \\
\text { hospital admissions; } \\
15 \% \text { required NG feedil }\end{array}$ \\
\hline $\begin{array}{l}\text { Rocks et al., } \\
2014^{10}\end{array}$ & $\begin{array}{l}\text { Cross- } \\
\text { Sectional } \\
\text { Study } \\
\text { (prospective) }\end{array}$ & Australia & $\begin{array}{l}\text { TF } \\
0(3)\end{array}$ & $\begin{array}{l}17 \\
(n / a)\end{array}$ & N/A & $\begin{array}{l}\text { Variety of } \\
\text { Settings }\end{array}$ & $\begin{array}{l}\text { Describe } \\
\text { practices of } \\
\text { Australian } \\
\text { dietitians in } \\
\text { management of } \\
\text { AN }\end{array}$ & $\begin{array}{l}\text { Secondary } \\
\text { (Inadequate } \\
\text { oral intake) }\end{array}$ & $\begin{array}{l}\text { All dietitians stated OR } \\
\text { was offered first with } \\
\text { supplementation. 82\% } \\
\text { recommended } \\
\text { implementing NG feed } \\
\text { as part of re-feeding } \\
\text { process. }\end{array}$ \\
\hline $\begin{array}{l}\text { Maginot et } \\
\text { al., } 2017^{18}\end{array}$ & $\begin{array}{l}\text { Cohort Study } \\
\text { (retrospective) }\end{array}$ & USA & TF 1 & $\begin{array}{l}87 \\
(73)\end{array}$ & $8-20$ & $\begin{array}{l}\text { Medical } \\
\text { Behavioural } \\
\text { Unit }\end{array}$ & $\begin{array}{l}\text { Safety of higher } \\
\text { calorie } \\
\text { nutritional } \\
\text { rehabilitation } \\
\text { protocol (NRP) }\end{array}$ & $\begin{array}{l}\text { Secondary } \\
\text { (Inadequate } \\
\text { oral intake) }\end{array}$ & $\begin{array}{l}\text { Lower \%IBW on admis } \\
\text { more important predict } \\
\text { of HP than initial calor } \\
\text { Malnourished patients } \\
\text { started on lower calori } \\
\text { more likely to have NG } \\
\text { tube. }\end{array}$ \\
\hline $\begin{array}{l}\text { Paccagnella } \\
\text { et al., } \\
2006^{20}\end{array}$ & $\begin{array}{l}\text { Cohort Study } \\
\text { (prospective) }\end{array}$ & Italy & TF 1 & $\begin{array}{l}24 \\
(24)\end{array}$ & $11-32$ & “Hospital” & $\begin{array}{l}\text { Define minimal } \\
\text { criteria for } \\
\text { "lifesaving" } \\
\text { treatment and } \\
\text { submit a patient } \\
\text { to NG }\end{array}$ & $\begin{array}{l}\text { Secondary } \\
\text { (medical } \\
\text { instability) }\end{array}$ & $\begin{array}{l}\text { Symptomatology impr } \\
\text { the day after NG; is } \\
\text { beneficial especially w } \\
\text { used for life saving } \\
\text { treatment initially }\end{array}$ \\
\hline $\begin{array}{l}\text { Silber et al., } \\
2004^{21}\end{array}$ & $\begin{array}{l}\text { Cohort Study } \\
\text { (retrospective) }\end{array}$ & USA & TF 10 & $\begin{array}{l}14 \\
(0)\end{array}$ & $12-18$ & $\begin{array}{l}\text { Adolescent } \\
\text { Inpatient } \\
\text { Unit }\end{array}$ & $\begin{array}{l}\text { Determine } \\
\text { outcomes of } \\
\text { supplementing } \\
\text { oral refeeding } \\
\text { with nocturnal } \\
\text { NG } \\
\text { supplementation }\end{array}$ & $\begin{array}{l}\text { Primary } \\
\text { (Routinely) }\end{array}$ & $\begin{array}{l}\text { Maximum kcals were } \\
\text { greater, weight achieve } \\
\text { discharge greater in } \\
\text { treatment group comp } \\
\text { to oral refeeding only }\end{array}$ \\
\hline $\begin{array}{l}\text { Madden et } \\
\text { al., } 2015^{22}\end{array}$ & $\begin{array}{l}\text { RCT } \\
\text { (prospective) }\end{array}$ & $\begin{array}{l}\text { Australia/ } \\
\text { USA }\end{array}$ & TF 3 & $\begin{array}{l}82 \\
(78)\end{array}$ & $12-18$ & $\begin{array}{l}\text { Paediatric } \\
\text { Medical } \\
\text { Ward }\end{array}$ & $\begin{array}{l}\text { Long term } \\
\text { outcomes of } \\
\text { treating to } \\
\text { restore weight } \\
\text { rather than just } \\
\text { to medically } \\
\text { stabilise }\end{array}$ & $\begin{array}{l}\text { Secondary } \\
\text { (Medical } \\
\text { instability) }\end{array}$ & $\begin{array}{l}\text { No difference in hospit } \\
\text { days used after initial } \\
\text { admission, total fewer } \\
\text { days in hospital to ach } \\
\text { medical stability. }\end{array}$ \\
\hline $\begin{array}{l}\text { Agostino et } \\
\text { al., } 2013^{23}\end{array}$ & $\begin{array}{l}\text { Cohort Study } \\
\text { (retrospective) }\end{array}$ & Canada & $\begin{array}{l}\text { TF } 8 \\
\text { FU } \\
0(6)\end{array}$ & $\begin{array}{l}165 \\
(158)\end{array}$ & $10-18$ & $\begin{array}{l}\text { Paediatric } \\
\text { Medical } \\
\text { Ward }\end{array}$ & $\begin{array}{l}\text { Difference in } \\
\text { LOS between } \\
\text { adolescent ED } \\
\text { treated with } \\
\text { short-term } \\
\text { continuous NG } \\
\text { feeding vs. } \\
\text { managed with } \\
\text { lower calorie } \\
\text { meals }\end{array}$ & $\begin{array}{l}\text { Primary } \\
\text { (Routinely) }\end{array}$ & $\begin{array}{l}\text { LOS reduced in the NG } \\
\text { cohort; No significant } \\
\text { difference in complica' } \\
\text { or electrolyte abnorma } \\
\text { ( } 90 \% \text { NG cohort receiv } \\
\text { prophylactic phosphat }\end{array}$ \\
\hline $\begin{array}{l}\text { Parker et al., } \\
2016^{24}\end{array}$ & $\begin{array}{l}\text { Cohort Study } \\
\text { (retrospective) }\end{array}$ & Australia & TF 3 & $\begin{array}{l}167 \\
(152)\end{array}$ & $14-19$ & $\begin{array}{l}\text { Adolescent } \\
\text { ED unit }\end{array}$ & $\begin{array}{l}\text { Weight gain and } \\
\text { complications } \\
\text { associated with } \\
\text { refeeding } \\
\text { prescribed } \\
\text { greater initial } \\
\text { calories }\end{array}$ & $\begin{array}{l}\text { Secondary } \\
\text { (Medical } \\
\text { instability) }\end{array}$ & $\begin{array}{l}\text { Mean starting intake } \mathrm{n} \\
2611.7 \mathrm{kcal} / \mathrm{day}(58.4 \\
\mathrm{kcal} / \mathrm{kg} \text { ) With inclusior } \\
\text { phosphate } \\
\text { supplementation no } \\
\text { increased risk of RS. }\end{array}$ \\
\hline $\begin{array}{l}\text { Madden et } \\
\text { al., } 2015^{25}\end{array}$ & $\begin{array}{l}\text { RCT } \\
\text { (prospective) }\end{array}$ & Australia & $\begin{array}{l}\text { TF } \\
1(9)\end{array}$ & $\begin{array}{l}78 \\
(74)\end{array}$ & $12-18$ & $\begin{array}{l}\text { Paediatric } \\
\text { ED service }\end{array}$ & $\begin{array}{l}\text { More rapid } \\
\text { refeeding } \\
\text { protocol } \\
\text { promotes initial } \\
\text { weight recovery } \\
\text { and medical } \\
\text { stability. }\end{array}$ & $\begin{array}{l}\text { Primary } \\
\text { (Medical } \\
\text { instability) }\end{array}$ & $\begin{array}{l}\text { Adequate weight gain } \\
\text { minimal adverse effec } \\
\text { were observed. All pati } \\
\text { gained weight in week } \\
\text { with no cases of HP or }\end{array}$ \\
\hline
\end{tabular}

Key: N = number of participants; FU = follow up; TF = Time Frame; NG = Nasogastric (Feeding); LOS = Length of Stay; ED = Eating disorder; EO = Early onset; AN nervosa; RS = refeeding syndrome; \% IBW = percentage ideal bodyweight; HP = hypophosphataemia; $\mathrm{OR}=$ oral refeeding; RCT = randomised control trial.

Appendix 2 Risk of Bias in eligible studies 


\begin{tabular}{|c|c|c|c|c|c|c|c|c|c|}
\hline $\begin{array}{l}\text { Study } \\
\text { Design }\end{array}$ & Country Set & $\begin{array}{l}\text { Time } \\
\text { Frame / } \\
\text { Follow up } \\
\text { years } \\
\text { (months) }\end{array}$ & $\begin{array}{l}N \text { total } \\
\text { (Female) }\end{array}$ & $\begin{array}{l}\text { Age } \\
\text { Range } \\
\text { (years) }\end{array}$ & Setting & Aims & $\begin{array}{l}\text { NG Primary/ } \\
\text { Secondary } \\
\text { Outcome? } \\
\text { (Reason for } \\
\text { Implementing } \\
\text { NG) }\end{array}$ & $\begin{array}{l}\text { Main } \\
\text { Outcomes }\end{array}$ & Risk of Bias \\
\hline $\begin{array}{l}\text { Kezelman et } \\
\text { al., } 2018^{26}\end{array}$ & $\begin{array}{l}\text { Cohort } \\
\text { (prospective) }\end{array}$ & Australia & $\begin{array}{l}\text { TF } 1(2) \\
\text { FU 8-66 } \\
\text { days }\end{array}$ & $\begin{array}{l}31 \\
(31)\end{array}$ & $15-19$ & $\begin{array}{l}\text { Specialist } \\
\text { ED } \\
\text { Adolescent } \\
\text { medical } \\
\text { ward }\end{array}$ & $\begin{array}{l}\text { Explore the } \\
\text { relationship } \\
\text { between anxiety } \\
\text { and weight } \\
\text { restoration }\end{array}$ & $\begin{array}{l}\text { Secondary } \\
\text { (Medical } \\
\text { instability) }\end{array}$ & $\begin{array}{l}\text { All patients received } N 1 \\
\text { initially. No establishec } \\
\text { relationship between } \\
\text { changes in anxiety anc } \\
\text { weight restoration. }\end{array}$ \\
\hline $\begin{array}{l}\text { Fuller et al., } \\
2019^{27}\end{array}$ & $\begin{array}{l}\text { Cross- } \\
\text { Sectional } \\
\text { Study } \\
\text { (prospective) }\end{array}$ & $\begin{array}{l}\text { UK/ } \\
\text { Ireland }\end{array}$ & TF 1 & $\begin{array}{l}134 \\
(n / a)\end{array}$ & $\mathrm{n} / \mathrm{a}$ & $\begin{array}{l}\text { Variety of } \\
\text { Settings }\end{array}$ & $\begin{array}{l}\text { Identify } \\
\text { common current } \\
\text { practice and if } \\
\text { specialist ED } \\
\text { units are } \\
\text { managing AN } \\
\text { differently to } \\
\text { other inpatient } \\
\text { settings }\end{array}$ & $\begin{array}{l}\text { Primary } \\
\text { (Inadequate } \\
\text { oral intake) }\end{array}$ & $\begin{array}{l}43.3 \% \text { reported that the } \\
\text { were able to facilitate } \\
\text { feeding; } \\
79 \% \text { of units providing } \\
\text { feeding were able to } \\
\text { facilitate physical } \\
\text { interventions }\end{array}$ \\
\hline $\begin{array}{l}\text { Street et al. } \\
2016^{28}\end{array}$ & $\begin{array}{l}\text { Case Reports } \\
\text { (prospective) }\end{array}$ & England & $\begin{array}{l}\text { TF } 3 \\
\text { FU 1-2 }\end{array}$ & $\begin{array}{l}31 \\
(30)\end{array}$ & $10-17$ & $\begin{array}{l}\text { Paediatric } \\
\text { medical } \\
\text { ward }\end{array}$ & $\begin{array}{l}\text { Evaluate joint } \\
\text { care ED pathway } \\
\text { between CAMHS } \\
\text { and paediatric } \\
\text { wards }\end{array}$ & $\begin{array}{l}\text { Secondary } \\
\text { (Medical } \\
\text { Instability) }\end{array}$ & $\begin{array}{l}\text { Time-limited admissio } \\
\text { with boundaried-care } \mathrm{r} \\
\text { are easier to manage } a \\
\text { enjoyed feeling suppor } \\
\text { by CAMHS }\end{array}$ \\
\hline $\begin{array}{l}\text { Couturier } \\
\text { and } \\
\text { Mahmood, } \\
2009^{29}\end{array}$ & $\begin{array}{l}\text { Cohort Study } \\
\text { (retrospective) }\end{array}$ & Canada & $\begin{array}{l}\text { TF } 2 \\
\text { FU } 1\end{array}$ & $\begin{array}{l}21 \\
(19)\end{array}$ & $11-17$ & $\begin{array}{l}\text { Psychiatric } \\
\text { Inpatient } \\
\text { Unit }\end{array}$ & $\begin{array}{l}\text { Understand } \\
\text { whether } \\
\text { implementing } \\
\text { meal support } \\
\text { therapy reduced } \\
\text { need for NG }\end{array}$ & $\begin{array}{l}\text { Primary } \\
\text { (Inadequate } \\
\text { oral intake) }\end{array}$ & $\begin{array}{l}\text { Meal support therapy } \\
\text { reduces need for } N G \\
(66.7-11.1 \% \text { after } \\
\text { implementation }(P<0 \text {. }\end{array}$ \\
\hline $\begin{array}{l}\text { Falcoski et } \\
\text { al.,2020 }\end{array}$ & $\begin{array}{l}\text { Case Series } \\
\text { (prospective) }\end{array}$ & UK & TF 1 & $\begin{array}{l}3 \\
(2)\end{array}$ & $11-14$ & $\begin{array}{l}\text { Specialist } \\
\text { ED unit }\end{array}$ & $\begin{array}{l}\text { Evaluate new } \\
\text { dietetic } \\
\text { guidelines for } \\
\text { AN in clinical } \\
\text { practice }\end{array}$ & $\begin{array}{l}\text { Primary } \\
\text { (variable) }\end{array}$ & $\begin{array}{l}\text { Different use of NG } \\
\text { feeding to suit individu } \\
\text { use of continuous and } \\
\text { single bolus feeds via } \\
\text { tube }\end{array}$ \\
\hline $\begin{array}{l}\text { O'Connor et } \\
\text { al., } 2016^{31}\end{array}$ & $\begin{array}{l}\text { RCT } \\
\text { (prospective) }\end{array}$ & UK & TF 2 & $\begin{array}{l}36 \\
(34)\end{array}$ & $10-16$ & $\begin{array}{l}\text { Paediatric } \\
\text { medical } \\
\text { Ward }\end{array}$ & $\begin{array}{l}\text { Higher calorie } \\
\text { refeeding } \\
\text { anthropometric } \\
\text { outcomes, } \\
\text { cardiac and } \\
\text { biochemical } \\
\text { markers }\end{array}$ & $\begin{array}{l}\text { Secondary } \\
\text { (Inadequate } \\
\text { oral intake) }\end{array}$ & $\begin{array}{l}\text { Adolescents on high } \\
\text { energy intake had grea } \\
\text { weight gain. } 11 \% \\
\text { participants required } \mathrm{N} \\
\text { feeding for failure to } \mathrm{n} \\
80 \% \text { oral intake. }\end{array}$ \\
\hline $\begin{array}{l}\text { Akgul et al., } \\
2016^{35}\end{array}$ & $\begin{array}{l}\text { Case Series } \\
\text { (retrospective) }\end{array}$ & Turkey & TF 4 & $\begin{array}{l}13 \\
(0)\end{array}$ & $11-17$ & $\begin{array}{l}\text { Paediatric } \\
\text { Medical } \\
\text { Ward }\end{array}$ & $\begin{array}{l}\text { Describe } \\
\text { medical, } \\
\text { psychiatric, } \\
\text { cultural features } \\
\text { of adolescent } \\
\text { males with an } \\
\text { ED }\end{array}$ & $\begin{array}{l}\text { Secondary } \\
\text { (Inadequate } \\
\text { oral intake) }\end{array}$ & $\begin{array}{l}\text { Male:female increased } \\
(3.6: 1 \mathrm{~F}: \mathrm{M}) ; 2 / 13 \text { given } \\
\text { due to refusal to eat in } \\
\text { hospital }\end{array}$ \\
\hline $\begin{array}{l}\text { Akgul et al., } \\
2016^{36}\end{array}$ & $\begin{array}{l}\text { Cohort Study } \\
\text { (retrospective) }\end{array}$ & Turkey & TF 6 & $\begin{array}{l}35 \\
(28)\end{array}$ & $11-17$ & $\begin{array}{l}\text { Paediatric } \\
\text { Medical } \\
\text { Ward }\end{array}$ & $\begin{array}{l}\text { Explore } \\
\text { paediatric unit } \\
\text { where no } \\
\text { specific ED unit } \\
\text { for to discuss } \\
\text { refeeding } \\
\text { approaches and } \\
\text { goals for } \\
\text { discharge }\end{array}$ & $\begin{array}{l}\text { Primary } \\
\text { (variable) }\end{array}$ & $\begin{array}{l}\text { Paediatric ward is } \\
\text { acceptable where } \\
\text { specialist ED inpatient } \\
\text { not viable; specialist uI } \\
\text { better however limited } \\
\text { resources }\end{array}$ \\
\hline $\begin{array}{l}\text { Nehring et } \\
\text { al., } 2014^{37}\end{array}$ & $\begin{array}{l}\text { Cohort Study } \\
\text { (retrospective) }\end{array}$ & Germany & $\begin{array}{l}\text { TF } 10 \\
\text { FU } \\
1-12\end{array}$ & $\begin{array}{l}208 \\
(208)\end{array}$ & $12-18$ & $\begin{array}{l}\text { Psychiatric } \\
\text { Inpatient } \\
\text { Unit }\end{array}$ & $\begin{array}{l}\text { Short-term and } \\
\text { long-term } \\
\text { outcomes of } \\
\text { treating with EN } \\
\text { compared to no } \\
\text { EN }\end{array}$ & $\begin{array}{l}\text { Primary } \\
\text { (not } \\
\text { discussed) }\end{array}$ & $\begin{array}{l}\text { No significant differen } \\
\text { recovery following NG; } \\
34 \% \text { had NG }\end{array}$ \\
\hline $\begin{array}{l}\text { Neiderman } \\
\text { et al., } \\
2004^{38}\end{array}$ & $\begin{array}{l}\text { Case reports } \\
\text { (prospective) }\end{array}$ & England & FU 1 & $\begin{array}{l}4 \\
(3)\end{array}$ & $13-16$ & $\begin{array}{l}\text { Adolescent } \\
\text { Unit }\end{array}$ & $\begin{array}{l}\text { Report of } \\
\text { gastrostomy or } \\
\text { jejunostomy use } \\
\text { in } 4 \text { cases of AN }\end{array}$ & $\begin{array}{l}\text { Secondary } \\
\text { (Medical } \\
\text { instability) }\end{array}$ & $\begin{array}{l}\text { 4/4 patients required } \uparrow \\
\text { feeding and progresse } \\
\text { require } \\
\text { gastrostomy/jejuonos } \\
\text { due to complications }\end{array}$ \\
\hline
\end{tabular}

Key: N = number of participants; FU = follow up; TF = Time Frame; NG = Nasogastric (Feeding); LOS = Length of Stay; ED = Eating disorder; EO = Early onset; AN nervosa; RS = refeeding syndrome; \% IBW = percentage ideal bodyweight; HP = hypophosphataemia; $\mathrm{OR}=$ oral refeeding; $\mathrm{RCT}=$ randomised control trial.

Appendix 2 Risk of Bias in eligible studies 


\begin{tabular}{|c|c|c|c|c|c|c|c|c|c|}
\hline $\begin{array}{l}\text { Study } \\
\text { Design }\end{array}$ & Country Set & $\begin{array}{l}\text { Time } \\
\text { Frame / } \\
\text { Follow up } \\
\text { years } \\
\text { (months) }\end{array}$ & $\begin{array}{l}N \text { total } \\
\text { (Female) }\end{array}$ & $\begin{array}{l}\text { Age } \\
\text { Range } \\
\text { (years) }\end{array}$ & Setting & Aims & $\begin{array}{l}\text { NG Primary/ } \\
\text { Secondary } \\
\text { Outcome? } \\
\text { (Reason for } \\
\text { Implementing } \\
\text { NG) }\end{array}$ & $\begin{array}{l}\text { Main } \\
\text { Outcomes }\end{array}$ & Risk of Bias \\
\hline $\begin{array}{l}\text { Robb et al., } \\
2002^{39}\end{array}$ & $\begin{array}{l}\text { Cohort Study } \\
\text { (retrospective) }\end{array}$ & USA & TF 6 & $\begin{array}{l}100 \\
(100)\end{array}$ & $12-18$ & $\begin{array}{l}\text { Paediatric } \\
\text { Medical } \\
\text { Ward }\end{array}$ & $\begin{array}{l}\text { Compare short- } \\
\text { term outcomes } \\
\text { of oral vs. } \\
\text { supplemental } \\
\text { nocturnal } \\
\text { nasogastric } \\
\text { refeeding }\end{array}$ & $\begin{array}{l}\text { Primary } \\
\text { (Routinely) }\end{array}$ & $\begin{array}{l}\text { Weight gain significan } \\
\text { increased in treatment } \\
\text { group, no significant } \\
\text { difference in length of } \\
\text { hospital stay }\end{array}$ \\
\hline $\begin{array}{l}\text { Neiderman } \\
\text { et al., } \\
2001^{40}\end{array}$ & $\begin{array}{l}\text { Cross- } \\
\text { Sectional } \\
\text { Study } \\
\text { (retrospective) }\end{array}$ & UK & $\begin{array}{l}\mathrm{TF} \\
1-18\end{array}$ & $\begin{array}{l}58(21 \\
\text { patients } \\
37 \\
\text { parents }) \\
(19 / 21)\end{array}$ & $\begin{array}{l}\text { Patients } \\
9-17 \text { at } \\
\text { start of } \\
\text { study }\end{array}$ & $\begin{array}{l}\text { Paediatric } \\
\text { Medical } \\
\text { Ward }\end{array}$ & $\begin{array}{l}\text { Analyse patient } \\
\text { and parent } \\
\text { views on NG } \\
\text { feeding }\end{array}$ & $\begin{array}{l}\text { Primary } \\
\text { (not } \\
\text { discussed) }\end{array}$ & $\begin{array}{l}71 \% \text { patients said they } \\
\text { not consent to NG feec } \\
\text { patients feared weight } \\
\text { and loss of control ove } \\
\text { calorie intake }\end{array}$ \\
\hline $\begin{array}{l}\text { Gusella et } \\
\text { al., } 2017^{41}\end{array}$ & $\begin{array}{l}\text { Cohort Study } \\
\text { (retrospective) }\end{array}$ & Canada & $\begin{array}{l}\text { TF } 13 \\
\text { FU } 1\end{array}$ & $\begin{array}{l}46 \\
(43)\end{array}$ & $9-15$ & $\begin{array}{l}\text { Outpatient } \\
\text { ED team }\end{array}$ & $\begin{array}{l}\text { Compare parent } \\
\text { led treatment } \\
\text { (PIC) to } \\
\text { conventional } \\
\text { treatment }\end{array}$ & $\begin{array}{l}\text { Secondary } \\
\text { (Medical } \\
\text { Instability) }\end{array}$ & $\begin{array}{l}\text { PIC had greater increas } \\
\% \text { IBW, fewer } \\
\text { hospitalisations, short' } \\
\text { admissions, less likely } \\
\text { receive NG feeding }\end{array}$ \\
\hline $\begin{array}{l}\text { Madden et } \\
\text { al., } 2009^{42}\end{array}$ & $\begin{array}{l}\text { Cross- } \\
\text { Sectional } \\
\text { Study } \\
\text { (prospective) }\end{array}$ & Australia & TF 3 & $\begin{array}{l}101 \\
(74)\end{array}$ & $5-13$ & $\begin{array}{l}\text { Medical } \\
\text { Ward and } \\
\text { Psychiatric } \\
\text { Inpatient } \\
\text { Wards }\end{array}$ & $\begin{array}{l}\text { Collect } \\
\text { epidemiological } \\
\text { data on EO-ED }\end{array}$ & $\begin{array}{l}\text { Secondary } \\
\text { (not } \\
\text { discussed) }\end{array}$ & $\begin{array}{l}\text { Most were hospitalisec } \\
\text { (78\%), mean duration ( } \\
\text { hospitalisation was } 24 \\
\text { days; } 58 \% \text { inpatients } \mathrm{N} \\
\text { tube fed. }\end{array}$ \\
\hline $\begin{array}{l}\text { van Noort et } \\
\text { al., } 2018^{43}\end{array}$ & $\begin{array}{l}\text { Cohort Study } \\
\text { (prospective) }\end{array}$ & Germany & TF 3 & $\begin{array}{l}120 \\
(120)\end{array}$ & $9-19$ & $\begin{array}{l}\text { Specialist } \\
\text { ED unit }\end{array}$ & $\begin{array}{l}\text { Evaluate } \\
\text { characteristics } \\
\text { of EO-AN } \\
\text { compared with } \\
\text { AO-AN. }\end{array}$ & $\begin{array}{l}\text { Secondary } \\
\text { (Inadequate } \\
\text { oral intake) }\end{array}$ & $\begin{array}{l}\text { NG tube feeding requir } \\
\text { more in EO-AN than AC } \\
\text { Restrictive more comm } \\
\text { in EO. }\end{array}$ \\
\hline $\begin{array}{l}\text { Strik Lievers } \\
\text { et al., } \\
2009^{44}\end{array}$ & $\begin{array}{l}\text { Cohort Study } \\
\text { (prospective) }\end{array}$ & France & TF 8 & $\begin{array}{l}213 \\
(213)\end{array}$ & $12-22$ & $\begin{array}{l}\text { Psychiatric } \\
\text { Ward }\end{array}$ & $\begin{array}{l}\text { Clinical } \\
\text { variables } \\
\text { influencing the } \\
\text { length of stay } \\
\text { (LOS) of } \\
\text { inpatient } \\
\text { treatment for AN }\end{array}$ & $\begin{array}{l}\text { Secondary } \\
\text { (Medical } \\
\text { instability) }\end{array}$ & $\begin{array}{l}\text { Requirement for tube } \\
\text { feeding was predictor } 1 \\
\text { LOS (longer) tube feed } \\
\text { required in } 27 \% \\
\text { admissions. }\end{array}$ \\
\hline $\begin{array}{l}\text { Halse et al., } \\
2005^{45}\end{array}$ & $\begin{array}{l}\text { Cross- } \\
\text { Sectional } \\
\text { Study } \\
\text { (prospective) }\end{array}$ & Australia & TF 1 & $\begin{array}{l}23 \\
(23)\end{array}$ & $12-20$ & $\begin{array}{l}\text { Adolescent } \\
\text { Medical } \\
\text { Ward }\end{array}$ & $\begin{array}{l}\text { Examine the } \\
\text { meanings that } \\
\text { patients } \\
\text { attached to NG }\end{array}$ & $\begin{array}{l}\text { Primary } \\
(\mathrm{N} / \mathrm{A})\end{array}$ & $\begin{array}{l}\text { Categories: unpleasan } \\
\text { physical experience, a } \\
\text { necessary intervention } \\
\text { physical and psycholo } \\
\text { signifier of AN, a focus } \\
\text { struggle for control. }\end{array}$ \\
\hline $\begin{array}{l}\text { Clausen et } \\
\text { al., } 2018^{46}\end{array}$ & $\begin{array}{l}\text { Cross- } \\
\text { Sectional } \\
\text { Study } \\
\text { (retrospective) }\end{array}$ & Denmark & TF 13 & $\begin{array}{l}4727 \\
(4387)\end{array}$ & $10-40+$ & $\begin{array}{l}\text { Psychiatric/ } \\
\text { Medical } \\
\text { Ward }\end{array}$ & $\begin{array}{l}\text { Frequency of } \\
\text { various } \\
\text { involuntary } \\
\text { measures in AN } \\
\text { patients }\end{array}$ & $\begin{array}{l}\text { Secondary } \\
\text { (not } \\
\text { discussed) }\end{array}$ & $\begin{array}{l}\text { Involuntary tube feedir } \\
\text { was most frequent } \\
\text { measure used. }\end{array}$ \\
\hline $\begin{array}{l}\text { Bayes and } \\
\text { Madden, } \\
2011^{47}\end{array}$ & $\begin{array}{l}\text { Case Series } \\
\text { (retrospective) }\end{array}$ & Australia & TF 2 & $\begin{array}{l}10 \\
(0)\end{array}$ & $10-13$ & $\begin{array}{l}\text { Paediatric } \\
\text { medical } \\
\text { Hospital }\end{array}$ & $\begin{array}{l}\text { Demographic } \\
\text { and clinical } \\
\text { features of male } \\
\text { inpatients with } \\
\text { EO ED }\end{array}$ & $\begin{array}{l}\text { Secondary } \\
\text { (Medical } \\
\text { instability) }\end{array}$ & $\begin{array}{l}\text { Only } 3 / 10 \text { participants } \\
\text { full criteria for AN; } 60 \% \\
\text { required NG feeding. }\end{array}$ \\
\hline $\begin{array}{l}\text { Kodua et } \\
\text { al.,2020 }\end{array}$ & $\begin{array}{l}\text { Case Reports } \\
\text { (prospective) }\end{array}$ & UK & TF 1 & $\begin{array}{l}8 \\
(n / a)\end{array}$ & $\mathrm{n} / \mathrm{a}$ & $\begin{array}{l}\text { ED } \\
\text { inpatient } \\
\text { units }\end{array}$ & $\begin{array}{l}\text { Nursing } \\
\text { assistants' } \\
\text { experiences of } \\
\text { manual } \\
\text { restraint for NG } \\
\text { feeding }\end{array}$ & $\begin{array}{l}\text { Primary } \\
\text { (N/A) }\end{array}$ & $\begin{array}{l}3 \text { primary themes were } \\
\text { gathered: an unpleasa } \\
\text { practice, importance of } \\
\text { coping, becoming } \\
\text { (de)sensitized to NG } \\
\text { feeding. }\end{array}$ \\
\hline
\end{tabular}

Key: N = number of participants; FU = follow up; TF = Time Frame; NG = Nasogastric (Feeding); LOS = Length of Stay; ED = Eating disorder; EO = Early onset; AN nervosa; RS = refeeding syndrome; \% IBW = percentage ideal bodyweight; HP = hypophosphataemia; OR = oral refeeding; RCT = randomised control trial.

Appendix 2 Risk of Bias in eligible studies

\section{$<$ Table 1>}

\section{Results}




\subsection{Prevalence and Epidemiology}

YP with ED requiring NG were often medically unstable on admission $9,18,20,22,23,25,39$ and NG feeding was implemented as standard practice. ${ }^{22,23,26,39}$ NG was also implemented due to acute refusal of food or inability to meet oral intake, without significant medical instability, in five studies. $9,10,18,31,43$ In 13 studies (3 high risk of bias $28,35,47$ ) in which NG was not implemented as standard protocol for all patients, the percentage of ED YP administered NG feeding in all contexts (due to medical instability or inadequate oral diet) varied between $6 \%-66 \% .9,18,29,31,36,37,41-44$

Two studies, ${ }^{37,43}$ found NG feeding was more likely to be required in: patients of a lower age at admission (14.3 years compared to 15.3 yrs old, $\mathrm{P}<0.05^{37}$ and $20 \%$ in early onset AN compared to $0 \%$ in adult onset AN $\mathrm{P}<0.05^{43}$ ). Clausen ${ }^{46}$ described NG as the most frequently used involuntary measure in psychiatric practice and is most commonly used in 15-17 year olds. Studies included both male and female patients, however, out of 25 patient focused studies, most had a female majority and 6 studies ${ }^{20,26,37,39,43,44}$ were conducted on female only cohorts. 2 studies ${ }^{21,47}$ examined male only cohorts but both were high risk of bias. 1 study ${ }^{39}$ included only Caucasian participants however the majority of studies were conducted in affluent, Caucasian majority countries; $31 \%$ of the studies included were set in Australia, 14\% in the USA, 10\% in Canada. There were no studies from Asia, South America or Africa. In Australian based studies, NG was given due to refusal of oral intake in 2 studies $^{9,10}$ as well as to treat medical instability. ${ }^{26}$ Globally studies from North America ${ }^{18,21,39,41}$ and Turkey ${ }^{36}$ focused on medical instability in YP with ED. In the UK, three studies described NG use during medical instability after oral intake was refused ${ }^{27,28,40}$ and one where oral intake was inadequate. ${ }^{31}$

\subsection{Reported Weight Gain}

Four studies reported weight gain primarily in the context of ED YP with medical instability. ${ }^{24,25,26,44} 2$ of these studies ${ }^{24,26}$ for the first $24-72$ hours started with continuous NG feeding, using higher than standard calorie protocols, 2400-3000 kcal per day prevented any initial drop in weight. Between admission and discharge, Parker et $\mathrm{al}^{24}$ reported a mean overall weight gain of $7.4 \mathrm{kgs}$, Kezelman $2018^{26}$ reported a mean overall increase of $3.04 \mathrm{~kg} / \mathrm{m}^{2} \mathrm{BMl}$; Madden et $\mathrm{al}^{25}$ reported a mean weight gain of $2.79 \mathrm{kgs}$ during medical instability using continuous NG feeding at 2400 kcals per day. Skrik Liever et al ${ }^{44}$ reported $27 \%$ required NG feeding and linked this to a faster weight gain but gave no information related to NG feeding protocols.

3 Studies reported weight gain in the context of inadequate oral intake ${ }^{9,18,39}$. Maginot et al, $2017^{18}$ and Whitelaw et al, $2010^{9}$ reported NG bolus feeding in $13.8 \%$ and $15 \%$ in order to supplement oral diet with a mean weight gain of $3.1 \mathrm{kgs}$ and $2.6 \mathrm{kgs}$ respectively but did not report if this was specific to NG feeding. Robb et al ${ }^{39}$ compared nocturnal NG feeding to supplement oral diet (maximum $3255 \mathrm{kcals} / \mathrm{d}$ ) with oral intake (max $2508 \mathrm{kcals} / \mathrm{d}$ ) reporting nocturnal NG feeding weight gain of $5.4 \mathrm{kgs}$ versus $2.4 \mathrm{kgs}$ in the oral diet only group.

1 Study reported on weight gain where NG is routinely started on all ED YP regardless of context. ${ }^{23}$ Agostino et al ${ }^{23}$ compared a higher calorie (1500$1800 \mathrm{kcal} / \mathrm{d}$ ) continuous NG fed cohort to lower calorie oral bolus cohort (1000-1200kcal/d, divided 6 times per day), results showed mean weight gain was greater in the continuous NG fed group ( $1.22 \mathrm{kgs}$ per week) than the oral bolus fed group ( $0.08 \mathrm{kgs}$ per week) over the first 2 weeks.

\subsection{Patient And Staff Experience Of Nasogastric Feeding}

Five studies used qualitative methods to analyse patient, parent and professional opinions on NG feeding. ${ }^{10,20,40,45,48} \mathrm{~A}$ survey of dietitians found $82 \%$ considered NG feeding a necessary procedure if oral diet is inadequate. ${ }^{10}$ Psychiatric nursing assistant's views centred around: NG being an unpleasant practice, becoming sensitized or desensitized, and the importance of developing coping mechanisms to manage the distress.

An Australian study ${ }^{45}$ (conducted in a paediatric unit) found YP viewed being NG fed as: an unpleasant experience, a necessary intervention, a psychological signifier of illness, and an emphasis in an underlying struggle for control. Some described NG feeds as easier than eating as it "disguised" the amount due to not swallowing; others felt it was a form of punishment for not gaining enough weight. Conversely the YP in Paccagnella and colleagues ${ }^{20}$ research stated NG was helpful, particularly initially when an oral diet was challenging to manage.

\subsection{Feeding Regime And Calorie Intake}

A variety of different feeding regimes were identified in this review which are summarised in Table 2 . Refeeding protocols daily calorie intake varied greatly between studies particularly as many studies were evaluating the outcome of higher calorie refeeding protocols. . $^{9,18,22,24,31}$ Most studies tailored the calorie requirements to the individual patient, accounting for initial weight for height percentage and signs of medical instability. The majority commenced on daily intake of less than $2000 \mathrm{kcal}$ and increased periodically. 
Table 2

Nasogastric Feeding Protocol and Complications Identified in Studies Included in this Systematic Review.

\begin{tabular}{|c|c|c|c|c|c|}
\hline Study & $\begin{array}{l}\text { Risk of } \\
\text { Bias }\end{array}$ & Setting & $\begin{array}{l}\text { Method and Reason for } \\
\text { Implementation of NG }\end{array}$ & Feeding Regime & Complications \\
\hline $\begin{array}{l}\text { Whitelaw et } \\
\text { al, } 2010^{9}\end{array}$ & Medium & $\begin{array}{l}\text { Medical } \\
\text { Ward }\end{array}$ & $\begin{array}{l}\text { Oral intake supplemented with } \\
\text { bolus NG feeding if oral RDI not } \\
\text { met }\end{array}$ & $\begin{array}{l}\text { Minimum of } 1900 \mathrm{kcals} \text { on day } 1 \text { and } \\
\text { increased by } 300 \mathrm{kcal} \text { per day }\end{array}$ & $\begin{array}{l}38 \% \text { developed HP. HP was } \\
\text { associated with lower } \\
\text { \%IBW on admission }\end{array}$ \\
\hline $\begin{array}{l}\text { Rocks et al, } \\
2014^{10}\end{array}$ & Medium & $\begin{array}{l}\text { MH and } \\
\text { Medical } \\
\text { Wards }\end{array}$ & $\begin{array}{l}\text { High energy supplements and NG } \\
\text { feeds were commonly used to } \\
\text { meet RDI. }\end{array}$ & $\begin{array}{l}\text { The initial calorie intake recommended was } \\
\text { between } 800-1750 \mathrm{kcals}\end{array}$ & Not discussed \\
\hline $\begin{array}{l}\text { Maginot et } \\
\text { al, } 2017^{18}\end{array}$ & Medium & $\begin{array}{l}\text { Medical } \\
\text { Ward }\end{array}$ & $\begin{array}{l}\text { Bolus NG feeds supplemental to } \\
\text { oral intake if RDI not met }\end{array}$ & $\begin{array}{l}\text { Average of } 1185 \mathrm{kcal} \text { average which } \\
\text { increased to an average of } 1781 \text { kcals (range } \\
1500-3000 \text { kcals) }\end{array}$ & $\begin{array}{l}\text { Hypomagnaemia and HP } \\
\text { reported, HP was more } \\
\text { likely in those under } 80 \% \\
\% \text { IBW }\end{array}$ \\
\hline $\begin{array}{l}\text { Paccagnella } \\
\text { et al, } 2006^{20}\end{array}$ & Medium & Unknown & $\begin{array}{l}\text { Continuous NG feeding until } \\
\text { medically stable }\end{array}$ & $\begin{array}{l}15.9-19.7 \mathrm{kcal} / \mathrm{kg} / \text { day; increased to } \\
30 \mathrm{kcal} / \mathrm{kg} / \mathrm{day} \text { after } 24 \text { hours. }\end{array}$ & $\begin{array}{l}\text { No patient developed } \\
\text { nausea, vomiting, or } \\
\text { worsened abdominal } \\
\text { symptoms; } 2 \text { developed } \\
\text { lower limb oedema despite } \\
\text { slow infusion. }\end{array}$ \\
\hline
\end{tabular}
Silber et al, High MH Ward $\begin{aligned} & \text { Routine nocturnal NG feeding to } \\ & \text { supplement daily oral intake vs }\end{aligned}$
$\mathbf{2 0 0 4 ^ { 2 1 }}$

$\begin{array}{lll}\text { Madden et } & \text { Low } & \begin{array}{l}\text { Medical } \\ \text { Ward }\end{array}\end{array}$

\begin{tabular}{|c|c|c|c|}
\hline $\begin{array}{l}\text { Agostino et } \\
\text { al, } 2013^{23}\end{array}$ & Medium & $\begin{array}{l}\text { Medical } \\
\text { Ward }\end{array}$ & $\begin{array}{l}\text { Routine continuous NG feeding at } \\
\text { a higher calorie intake compared } \\
\text { to lower calorie standard oral } \\
\text { intake. }\end{array}$ \\
\hline
\end{tabular}
oral refeeding only (control)

Continuous NG feeding until medically stable; followed by oral intake with supplemental nocturnal NG feeding until biomarkers stabilised.

Nocturnal NG feeding regime patients were prescribed calories individually ( $\max$ $4350 \mathrm{kcal})$ and 3400 in the oral refeeding group (control).

NG feeding continuously for $1-2$ days. Weight gain aim for $1 \mathrm{~kg}$ per week. Weaning to oral diet occurred as soon as medically stable - average 14 days on NG with feed of $2400-3000 \mathrm{kcal}$ per day increased by $200 \mathrm{kcal} /$ day vs. oral diet of $800-1200 \mathrm{kcal}$ increased by $150 \mathrm{kcal} /$ day. NG fed for 7 days then weaned over 3 days with $\mathrm{kcal}$ via NG reducing as meals replaced
Starting range for NG cohort $1200-2000 \mathrm{kcal}$

Epistaxis, nasal irritation.

Not discussed

Oral cohort $51 \%$ lost weight initially compared to $6 \%$ in the NG high kcal cohort. 2 cases of

Hypokalaemia (although both were abusing laxatives), HP.

Peripheral oedema (4\%) hypomagnaemia $(7 \%)$, hypokalaemia (2\%), HP (1\%). No incidence of RS or delirium. supplemental overnight NG feeding, or oral intake alone.

Start feed $2400 \mathrm{kcal}$ increasing to 2400 $3400 \mathrm{kcal} /$ day at $100 \mathrm{ml}$ per hour

Stated none developed RS or HP

$2400-3000 \mathrm{kcal}$ to meet weekly target of weight gain of $1 \mathrm{~kg} /$ week. In the first week average weight gain was $2.79 \mathrm{~kg}$. medically stable; followed by ora intake with supplemental nocturnal NG feeding until biomarkers stabilised. Average $\%$ IBW at initiation was 78

$\begin{array}{lll}\begin{array}{l}\text { Madden et } \\ \text { al, 2015 }\end{array} \text { Low } & \begin{array}{l}\text { Medical } \\ \text { Ward }\end{array} & \begin{array}{l}\text { Continuous NG feeding until } \\ \text { medically stable; followed by oral } \\ \text { intake with supplemental } \\ \text { nocturnal NG feeding until } \\ \text { biomarkers stabilised. Average } \\ \text { \%IBW at initiation was 78 }\end{array}\end{array}$

Kezelman et Medium Medical Continuous NG until medically
al $2018^{26} \quad$ Ward stable followed by oral intake supplemented by nocturnal NG feeding

$2400 \mathrm{kcal} /$ day for $24 \mathrm{hrs}$ or until medically stable, changed to oral diet starting $1800 \mathrm{kcal}$ increasing to a maximum of $3800 \mathrm{kcal}$ with nocturnal NG top up feeds stopped when $\mathrm{BMI}>18.5$

Fuller et al, Medium MH Ward Results from questionnaire $2019^{27} \quad$ showed non-specialist psychiatric units gave $73 \%$ NG as syringe bolus, $27 \%$ as enteral pump. Specialist ED units gave $85 \%$ as syringe bolus, $15 \%$ as enteral pump.

Volume of bolus feed ranged from 330 $1000 \mathrm{ml}$ average $564 \mathrm{ml}$ per feed. Bolus feed time ranged between $10-40$ minutes average being 20 minutes. If delivered by pump it was $>1$ hour.

Not discussed

NG feeds were higher in calories than meals to motivate eating.

Not discussed unstable and oral intake not me

Bolus NG feeding if patient failed to gain $1 \mathrm{~kg} /$ week or acute refusal of meals
Not discussed
Not discussed

Nausea, odynophagia, self-harm, epistaxis, anxiety, sadness, $38.4 \%$ patients experienced mild HP

Distress described during the procedure requiring Lorazepam
Starting feed $1200 \mathrm{kcal}$, increased by $200 \mathrm{kcal}$ per day to $2000 \mathrm{kcal} .1 \mathrm{NG}$ feed per day under restraint. Also described 1 bolus feed of $2000 \mathrm{kcal}$ due to no oral intake for 20 hours bolus NG feeds, single bolus of high calorie NG feeding, and 3 smaller single boluses. 


\begin{tabular}{|c|c|c|c|c|c|}
\hline Study & $\begin{array}{l}\text { Risk of } \\
\text { Bias }\end{array}$ & Setting & $\begin{array}{l}\text { Method and Reason for } \\
\text { Implementation of NG }\end{array}$ & Feeding Regime & Complications \\
\hline $\begin{array}{l}\text { O'Connor et } \\
\text { al, } 2016^{31}\end{array}$ & Low & $\begin{array}{l}\text { Medical } \\
\text { Ward }\end{array}$ & $\begin{array}{l}\text { Supplemental bolus NG feeding if } \\
\text { patients failed to meet } 80 \% \text { RDI. At } \\
\text { initiation \%IBW was }<78 \%\end{array}$ & $\begin{array}{l}\text { Compared 500kcal starting diet with } \\
1200 \mathrm{kcal}\end{array}$ & $\mathrm{HP}(28 \%)$ \\
\hline $\begin{array}{l}\text { Akgul et al, } \\
2016^{35}\end{array}$ & High & MH Ward & Not discussed & $\begin{array}{l}\text { Initiated at } 750 \mathrm{kcal} \text { per day and increased by } \\
220 \mathrm{kcal} \text { per day }\end{array}$ & $\begin{array}{l}\text { HP described in } 2 \text { cases } \\
\text { (unable to determine if this } \\
\text { was in those requiring NG) }\end{array}$ \\
\hline $\begin{array}{l}\text { Akgul et al, } \\
2016^{36}\end{array}$ & Medium & $\begin{array}{l}\text { Medical } \\
\text { Ward }\end{array}$ & $\begin{array}{l}\text { Not discussed, the majority of } \\
\text { young people were under } 80 \% \\
\% \text { IBW }\end{array}$ & $\begin{array}{l}\text { Started on an average of } 975 \mathrm{kcal} \text {. Average } \\
\text { duration of NG was } 2.5 \text { days }\end{array}$ & $\begin{array}{l}\text { HP described in } 2 \text { cases } \\
\text { (not stated if this was in } \\
\text { those requiring NG) }\end{array}$ \\
\hline $\begin{array}{l}\text { Robb et al, } \\
2002^{39}\end{array}$ & Medium & $\begin{array}{l}\text { Medical } \\
\text { Ward }\end{array}$ & $\begin{array}{l}\text { Nocturnal NG feeding to } \\
\text { supplement daily oral intake } \\
\text { during medical instability }\end{array}$ & $\begin{array}{l}\text { Starting NG feed at } 600 \text { kcal. Ratio oral kcal } \\
\text { to NG was approximately } 2: 1 \text {. NG feed via } \\
\text { pump at } 40 \text { cc per hour for } 4 \text { hours then } 60 \\
\text { cc per hour for } 4 \text { hours. Increases to } \\
1200 \mathrm{kcal} \text { NG feed over } 3 \text { nights. Weaned } \\
\text { when the young person is } 95 \% \text { IBW. }\end{array}$ & $\begin{array}{l}\text { Epistaxis }(11.5 \%) \text {, anxiety } \\
(3.8 \%) \text { treated with } \\
\text { Lorazepam, removal of NG } \\
\text { tube }(5.8 \%) \text {, nasal irritation } \\
(28.8 \%) .\end{array}$ \\
\hline $\begin{array}{l}\text { Neiderman } \\
\text { et al, } 2001^{40}\end{array}$ & High & $\begin{array}{l}\text { Medical } \\
\text { Ward }\end{array}$ & Not discussed & Not discussed & Removal of tube (55\%). \\
\hline
\end{tabular}

No study discussed in detail the strategy used to transition from NG feeds back to an oral diet. Those studies where NG was used for medical stabilisation often described a short period of NG before a quick transition back to an oral diet. ${ }^{22,23,36}$ In studies where continuous NG was provided, YP were sometimes not given the option of an oral diet so that their calorie intake could be closely monitored. ${ }^{22-24,31}$ These studies discussed ceasing NG feeds after the risk of RS had reduced; most gave a time frame between 2-14 days. ${ }^{24,44}$ Studies using bolus feeds stated that oral intake was encouraged and it was only when this was not fully achieved that supplementary NG was used. ${ }^{39}$ This appeared to be either after each meal, at set times during the day or once in the evening. ${ }^{27}$ For nocturnal feeds, oral diet was encouraged during the day. In most studies the NG feed supplemented any deficit in oral intake but occasionally also provided additional calories above those prescribed in the oral meal plan. ${ }^{22,25,39}$

\section{<Table 2>}

3.5 Length Of Time Receiving Ng Feeding

There was a wide variety in length of time receiving NG for medical instability. Agostino and colleagues ${ }^{23}$ delivered nutrition on a medical ward solely via NG for 14 days before commencing oral diet in addition to NG feeding. The average length of time on NG feeding in this study was 20.7 days; NG was terminated as YP accepted more than $50 \%$ oral caloric quota compared to theoretical reported quota. Madden et al ${ }^{22}$ RCT determined the duration of NG feeding was a minimum of 14 days, using biochemical markers of medical instability in a hospital setting. Conversely, Akgul and colleagues ${ }^{36}$ described a much shorter average time, 2.5 days, that YP required NG before transitioning to an oral diet. Conversely, in MH wards, if NG has to be given under restraint, it may be required for a significant duration; in one study ${ }^{46}$ the average was 170 days. Neiderman et al ${ }^{40}$ qualitative study describes patients time receiving $N G$ varying from 1 to 476 days (methods not explained).

2 studies examined therapeutic interventions to reduce the need for NG or length of time on it in medically stable YP. ${ }^{29,41}$ Couturier and Mahmood ${ }^{29}$ highlighted that meal support therapy reduced the requirement for NG feeding from $66.7-11.1 \%$, criteria for NG feeding was the same in both groups throughout and oral intake was encouraged. Gusella and colleagues ${ }^{41}$ compared parent led therapy (PLT) to non-specific therapy (psychologist led talking therapy). PLT was based on FBT and included parents reducing child exercise and increasing oral intake. Results demonstrated that YP receiving PLT had a significantly reduced requirement for NG $(P<0.05)$.

\subsection{Complications Associated With Ng Feeding}

Complications associated with NG feeding found in this review are summarised in Table 2, with the most frequently described being nasal irritation or epistaxis, anxiety related to the procedure and electrolyte disturbance (which occurred with both oral and NG refeeding). Overall, this review found 5 studies $^{9,18,23,24,29}$ reported some incidence of electrolyte disturbance, 2 studies ${ }^{29,39}$ described epistaxis and 1 study ${ }^{39}$ described behavioural problems associated with the procedure. A number of YP in MH wards required restraint to NG feed with one study reporting this was required for $66 \%$ of YP. ${ }^{24}$ NG under restraint was described as causing distress and risk of injury to both staff and YP. ${ }^{48}$ No study reported a YP developed RS. Nehring and colleagues ${ }^{37}$ concluded that NG feeding had no impact on growth, recovery or development of psychiatric co-morbidities.

Kezelman and colleagues (Australia) $2018^{26}$ assessed the impact on anxiety, depression and ED symptoms when using NG in adjunct to oral intake as part of a rapid refeeding regime. Changes in these symptoms were not attributed to the rate of weight restoration suggesting a rapid refeeding schedule would not exacerbate psychiatric symptoms.

\subsection{Length Of Stay Associated With Ng Feeding}


Length of stay was reported in studies from medical and $\mathrm{MH}$ ward settings, however, the specific package of treatment YP received in each study was different depending on the country of origin. For example, in Australian studies medical wards tended to include high levels of psychiatric treatment alongside medical treatment. ${ }^{26}$ Agostino and colleagues ${ }^{23}$ demonstrated that YP on medical wards having NG feeds had a mean LOS of 33.8 days compared to those in the same setting having an oral diet who had a mean of 50.9 days, however, the oral diet was lower in calories therefore taking longer for weight recovery and medical stabilisation. Conversely any hospital admission was significantly longer $(P<0.0001)$ for a YP requiring NG feeding compared to those managing an oral diet in a German retrospective cohort study. ${ }^{37}$ However, this study does not discuss the reasons NG was implemented. Maginot et al study ${ }^{18}$ in a medical ward (where NG was implemented due to insufficient oral intake) discussed NG feeding in the context of YP with more severe medical problems, (such as intractable vomiting and superior mesenteric artery syndrome) which therefore took longer to transition to oral diet, resulting in a longer admission.

Strik Lievers and colleagues ${ }^{44}$ concluded that, amongst others, requirement for NG feeding when NG was implemented due to medical instability was a factor affecting LOS on a psychiatric ward. In this study the mean LOS was significantly increased: 117 days for YP managing oral intake compared to 180 days for those requiring NG. They concluded that the requirement for NG was an indication of severity and resistance to oral feeding. ${ }^{44}$

\section{Discussion}

It is evident that there is a wide variety of practices regarding implementation and regime of NG feeding in YP with eating disorders globally. ${ }^{9}$ Given that the procedure can be painful ${ }^{48}$ for YP and cause complications ${ }^{29,39}$, there is an urgent need for research exploring this wide variation in use of NG feeding to enable future direction and best practice guidance clinicians. A review conducted by Rizzo and colleagues ${ }^{49}$ (2019), which focused on NG for acute refeeding, also found a wide variety of practices.

From this systematic review 3 methods of NG feeding in YP with ED were found: continuous, ${ }^{23,25}$ nocturnal, ${ }^{26,29}$ and bolus meal replacement. ${ }^{9}$ It is not possible from this review to discern the advantages and disadvantages of each method as no study made a direct comparison. When NG feeding is used under restraint bolus feeds are preferred due to concerns around the tube being removed by the YP once restraint had ceased. ${ }^{45}$ The main disadvantage to bolus feeding, in medically stable YP, is that the NG tube requires reinsertion each time a feed is required, however, it provides a tangible motivation to eat the full meal plan provided which, in practice, should always be encouraged over NG feeding in order to promote patient wellbeing. Further research is required to assess which method is the safest, most efficacious and best aids transition back to a fully oral diet.

Medical wards used continuous feeding more frequently than $\mathrm{MH}$ wards, however this tended to be for a short period of time while the YP was medically unstable, after this they would be transitioned to an oral diet. ${ }^{22,23,25,26}$ It is probable that medical wards primarily manage YP for short periods to stabilise acute physical health deterioration, while $\mathrm{MH}$ wards admit relatively medically stable YP and seek primarily to treat psychological ED symptoms that are preventing an adequate oral diet. This difference could account for the divergent outcomes from studies on the impact NG has on the LOS between medical and psychiatric settings. ${ }^{23,44}$

Similar to the review conducted by Hale and Logomarsino ${ }^{33}$ who found RS to be a rare complication, it is reassuring to find that no study in this review reported YP developing RS despite some studies starting on high calorie NG feeding plans. ${ }^{9,18,24,42}$ Although complications such as electrolyte abnormalities did occur there was no evidence that this was attributable to the NG feeding compared to oral diet. ${ }^{9,18,23,24}$ The results of this review support the conclusions from Rizzo and colleagues ${ }^{49}$ (2019) that NG feeds can be safely administered and have the advantage of shortening LOS when used to increase total caloric intake. However, further research is required to assess the optimum NG feeding regime for YP at different levels of RS risk. In two studies intensive meal support and concurrent therapy reduced the number of NG episodes (in medically stable YP) before managing a full oral diet. ${ }^{29,41}$ This could have the advantage of reducing LOS in medically stable YP.

There are a number of limitations to the conclusions that can be drawn from this review. The majority of studies included were retrospective and based around case note reviews which are subjective and therefore likely to be biased. A retrospective design also creates selection bias as those lost to follow up are not considered. Bias can also occur due to the different treatment groups being recorded at different times thus confounding variables may include different staff working at the setting and therefore different methods of treating YP. Only $52 \%$ of studies were conducted prospectively. Three studies were qualitative interview studies, examining patient or staff feelings towards NG feeding in practice which increases the risk of confirmation bias. The majority also had a relatively small sample size, again introducing the possibility of bias and reducing generalizability. $58 \%$ of the studies included only examined the effect of NG feeding as a secondary outcome of their study. It is not possible from these studies to make any comparison between NG feeding and oral intake due to the confounding effect that for the vast majority of studies only high risk, medically unstable YP were considered for NG feeding. Pragmatic, prospective studies that control for this confounder are required for any such comparison to be made.

\section{Conclusions}

This review describes the large differences in the use of NG for YP with ED in medical and psychiatric wards in a number of countries globally. NG feeding is an important aspect of treatment for YP with ED who are medically unstable and/or unable to manage an adequate oral diet. Although there are some RCT's examining aspects of NG use in YP with ED the majority of studies were retrospective cohorts or case series. There is a need for more high quality data in when to initiate NG, comparing different methods of delivering NG feeds and transitioning from NG to oral diet in YP with restrictive ED to enable future direction for clinicians.

\section{Abbreviations}


\%IBW percentage ideal body weight

AN anorexia nervosa

AOED adolescent onset eating disorder

CAMHS child and adolescent mental health service

ED eating disorder

EOED early onset eating disorder

FBT family-based therapy

HP hypophosphataemia

LOS length of stay

MH mental health

NG nasogastric feeding

OT occupational therapist

PLT parent led therapy

PRISMA Preferred Reporting Items for Systematic Reviews and Meta-Analyses

$\mathrm{RDI}$ recommended daily intake

RS refeeding syndrome

YP young person/people

\section{Appendix 1}

1. Naso-gastric or nasogastric or *enteric or *enteral or tube

2. (Anorexia or bulimia or eat* or feed*) NOT bowel NOT surgery NOT intestin*

3. (child* or paed* or adolescen* or teen* or young) NOT baby NOT toddler NOT infant NOT animal NOT maternal NOT parental NOT learning disabl* NOT learning disabil*

4. 1 AND 2 AND 3

\section{Appendix 2}

Risk of Bias in eligible studies 


\begin{tabular}{|c|c|c|c|c|c|c|c|c|c|c|}
\hline Authors & $\begin{array}{l}\text { Study } \\
\text { design }\end{array}$ & $\begin{array}{l}\text { Sample } \\
\text { size }\end{array}$ & $\begin{array}{l}\text { Unbiased } \\
\text { cohort } \\
\text { selection }\end{array}$ & $\begin{array}{l}\text { Selection } \\
\text { minimizes } \\
\text { baseline } \\
\text { differences in } \\
\text { demographic } \\
\text { factors }\end{array}$ & $\begin{array}{l}\text { Sample } \\
\text { size } \\
\text { calculated }\end{array}$ & $\begin{array}{l}\text { Validated } \\
\text { method for } \\
\text { ascertaining } \\
\text { clinical status } \\
\text { or participant } \\
\text { group }\end{array}$ & $\begin{array}{l}\text { Validated } \\
\text { methods for } \\
\text { assessing } \\
\text { variables of } \\
\text { interest }\end{array}$ & $\begin{array}{l}\text { Validated } \\
\text { methods } \\
\text { for } \\
\text { assessing } \\
\text { outcome }\end{array}$ & $\begin{array}{l}\text { Blind } \\
\text { outcome } \\
\text { assessment }\end{array}$ & Score \\
\hline $\begin{array}{l}\text { Whitelaw et } \\
\text { al., } 2010^{9}\end{array}$ & 2 & 2 & 0 & 0 & 0 & 6 & 6 & 6 & 0 & 22 \\
\hline $\begin{array}{l}\text { Rocks et al., } \\
2014^{10}\end{array}$ & 4 & 2 & 2 & 2 & 0 & 6 & 6 & 6 & 0 & 28 \\
\hline $\begin{array}{l}\text { Maginot et } \\
\text { al., } 2017^{18}\end{array}$ & 2 & 4 & 2 & 2 & 0 & 6 & 6 & 4 & 0 & 26 \\
\hline $\begin{array}{l}\text { Paccagnella } \\
\text { et al., } \\
2006^{20}\end{array}$ & 4 & 2 & 0 & 2 & 0 & 6 & 6 & 6 & 0 & 26 \\
\hline $\begin{array}{l}\text { Silber et al., } \\
2004^{21}\end{array}$ & 2 & 2 & 0 & 0 & 0 & 6 & 6 & 4 & 0 & 20 \\
\hline $\begin{array}{l}\text { Madden et } \\
\text { al., 2015b22 }\end{array}$ & 6 & 4 & 4 & 4 & 0 & 6 & 6 & 6 & 6 & 42 \\
\hline $\begin{array}{l}\text { Agostino et } \\
\text { al., } 2013^{23}\end{array}$ & 2 & 6 & 0 & 0 & 6 & 6 & 6 & 6 & 6 & 38 \\
\hline $\begin{array}{l}\text { Parker et al., } \\
2016^{24}\end{array}$ & 2 & 6 & 6 & 0 & 0 & 6 & 6 & 6 & 0 & 32 \\
\hline $\begin{array}{l}\text { Madden et } \\
\text { al., } 2015 a^{25}\end{array}$ & 6 & 4 & 6 & 4 & 6 & 6 & 6 & 6 & 6 & 50 \\
\hline $\begin{array}{l}\text { Kezelman et } \\
\text { al } 2018^{26}\end{array}$ & 4 & 2 & 2 & 2 & 0 & 6 & 6 & 6 & 0 & 28 \\
\hline $\begin{array}{l}\text { Fuller et al., } \\
2019^{27}\end{array}$ & 4 & 6 & 6 & 6 & 0 & 6 & 6 & 6 & 0 & 40 \\
\hline $\begin{array}{l}\text { Street et al., } \\
2016^{28}\end{array}$ & 0 & 2 & 0 & 0 & 0 & 0 & 0 & 6 & 0 & 8 \\
\hline $\begin{array}{l}\text { Couturier } \\
\text { and } \\
\text { Mahmood, } \\
2009^{29}\end{array}$ & 2 & 2 & 0 & 0 & 0 & 6 & 6 & 6 & 0 & 22 \\
\hline $\begin{array}{l}\text { O'Connor et } \\
\text { al., } 2016^{31}\end{array}$ & 6 & 2 & 4 & 4 & 6 & 6 & 6 & 6 & 6 & 46 \\
\hline $\begin{array}{l}\text { Falcoski et } \\
\text { al. } 2020^{30}\end{array}$ & 0 & 0 & 0 & 0 & 0 & 6 & 6 & 6 & 0 & 18 \\
\hline $\begin{array}{l}\text { Akgul et al., } \\
2016 a^{35}\end{array}$ & 0 & 2 & 0 & 0 & 0 & 6 & 0 & 0 & 0 & 8 \\
\hline $\begin{array}{l}\text { Akgul et al., } \\
2016 b^{36}\end{array}$ & 2 & 2 & 0 & 0 & 0 & 6 & 6 & 6 & 0 & 22 \\
\hline $\begin{array}{l}\text { Nehring et } \\
\text { al., } 2014^{37}\end{array}$ & 2 & 6 & 4 & 2 & 6 & 6 & 6 & 6 & 0 & 38 \\
\hline $\begin{array}{l}\text { Neiderman } \\
\text { et al., } \\
2000^{38}\end{array}$ & 0 & 0 & 0 & 0 & 0 & 6 & 6 & 4 & 0 & 16 \\
\hline $\begin{array}{l}\text { Robb et al., } \\
2002^{39}\end{array}$ & 4 & 6 & 4 & 4 & 0 & 6 & 6 & 6 & 0 & 36 \\
\hline $\begin{array}{l}\text { Neiderman } \\
\text { et al., } \\
2001^{40}\end{array}$ & 4 & 2 & 0 & 0 & 0 & 6 & 0 & 0 & 0 & 12 \\
\hline $\begin{array}{l}\text { Gusella et } \\
\text { al., } 2017^{41}\end{array}$ & 2 & 2 & 0 & 0 & 0 & 6 & 6 & 6 & 0 & 22 \\
\hline
\end{tabular}




\begin{tabular}{|c|c|c|c|c|c|c|c|c|c|c|}
\hline Authors & $\begin{array}{l}\text { Study } \\
\text { design }\end{array}$ & $\begin{array}{l}\text { Sample } \\
\text { size }\end{array}$ & $\begin{array}{l}\text { Unbiased } \\
\text { cohort } \\
\text { selection }\end{array}$ & $\begin{array}{l}\text { Selection } \\
\text { minimizes } \\
\text { baseline } \\
\text { differences in } \\
\text { demographic } \\
\text { factors }\end{array}$ & $\begin{array}{l}\text { Sample } \\
\text { size } \\
\text { calculated }\end{array}$ & $\begin{array}{l}\text { Validated } \\
\text { method for } \\
\text { ascertaining } \\
\text { clinical status } \\
\text { or participant } \\
\text { group }\end{array}$ & $\begin{array}{l}\text { Validated } \\
\text { methods for } \\
\text { assessing } \\
\text { variables of } \\
\text { interest }\end{array}$ & $\begin{array}{l}\text { Validated } \\
\text { methods } \\
\text { for } \\
\text { assessing } \\
\text { outcome }\end{array}$ & $\begin{array}{l}\text { Blind } \\
\text { outcome } \\
\text { assessment }\end{array}$ & Score \\
\hline $\begin{array}{l}\text { Madden et } \\
\text { al., } 2009^{42}\end{array}$ & 4 & 6 & 6 & 6 & 0 & 6 & 0 & 0 & 0 & 28 \\
\hline $\begin{array}{l}\text { van Noort et } \\
\text { al., } 2018^{43}\end{array}$ & 4 & 6 & 4 & 4 & 0 & 6 & 6 & 6 & 0 & 36 \\
\hline $\begin{array}{l}\text { Strik Lievers } \\
\text { et al., } \\
2009^{44}\end{array}$ & 4 & 6 & 4 & 4 & 0 & 6 & 6 & 6 & 0 & 36 \\
\hline $\begin{array}{l}\text { Halse et al., } \\
2005^{45}\end{array}$ & 4 & 2 & 2 & 2 & 0 & 6 & 6 & 6 & 0 & 28 \\
\hline $\begin{array}{l}\text { Clausen et } \\
\text { al., } 2018^{46}\end{array}$ & 4 & 6 & 6 & 6 & 2 & 6 & 6 & 6 & 0 & 42 \\
\hline $\begin{array}{l}\text { Bayes and } \\
\text { Madden, } \\
2011^{47}\end{array}$ & 0 & 2 & 2 & 0 & 0 & 6 & 2 & 4 & 0 & 16 \\
\hline $\begin{array}{l}\text { Kodua et } \\
\text { al. } 2020^{48}\end{array}$ & 0 & 0 & 0 & 0 & 0 & 6 & 6 & 6 & 0 & 18 \\
\hline $\begin{array}{l}\text { Key: Study d } \\
\text { Other column } \\
\text { risk }\end{array}$ & $\begin{array}{l}\text { n: RCT } \\
\text { lear e }\end{array}$ & $\begin{array}{l}\text { Prosp } \\
\text { nce }=6\end{array}$ & $\begin{array}{l}\text { e/cross s } \\
\text { ne eviden }\end{array}$ & $\begin{array}{l}\text { on }=4 \text { Retrosp } \\
=4 \text { Little evide }\end{array}$ & $\begin{array}{l}\text { e cohort }= \\
2 \text { No evide }\end{array}$ & $\begin{array}{l}\text { ase series }=0 \\
\mathrm{e}=0 ., \text { Total } \mathrm{sc}\end{array}$ & $\begin{array}{l}\text { le size: }>100 \\
-20=\text { High r }\end{array}$ & $\begin{array}{l}6,50-100 \\
21-40=N\end{array}$ & $\begin{array}{l}10-50=2,<1 \\
\text { ium risk }>40=\end{array}$ & $\begin{array}{l}=0 . \\
\text { ow }\end{array}$ \\
\hline
\end{tabular}

\section{Declarations}

Ethical Approval and Consent to Participate

No ethical approval or consent to participate required due to the nature of the study.

Consent for Publication:

All authors have reviewed the document and consent to publication.

\section{Availability of Supporting Data}

All articles analysed in this study can be found in Table 1 and can be traced back to primary articles using References on Page 16.

Competing Interests:

Authors declare no competing interests.

\section{Funding:}

No funding.

\section{Authors Contributions:}

$\mathrm{KH}$ and $\mathrm{CF}$ performed search of databases and created the document. All authors assessed bias risk. KH gathered data and interpreted results. $\mathrm{CF}$ performed the discussion. JM was responsible for references and editing.

\section{Acknowledgements:}

There are no other acknowledgements to be made.

\section{References}

1. National Guideline Alliance (UK). Eating Disorders: Recognition and Treatment. London: National Institute for Health and Care Excellence (UK); 2017 May. (NICE Guideline, No. 69.) Available from: https://www.ncbi.nlm.nih.gov/books/NBK436876/. 
2. Hay AP, Chinn D, Forbes D, Forbes S, Madden R, Newton, et al. Royal Australian and New Zealand College of Psychiatrists clinical practice guidelines for the treatment of eating disorders. Aust N Z J Psychiatry. 2014;48 11:977-1008.

3. Petkova BH, Simic M, Nicholls D, Ford T, Prina AM, Stuart R. Incidence of anorexia nervosa in young people in the UK and Ireland: a national surveillance study. BMJ Open. 2019;9:e027339. doi:10.1136/bmjopen-2018-027339.

4. Roux H, Chapelon E, Godart N. Epidemiology of anorexia nervosa: a review. Encephalitis. 2013;39(2):85-93.

5. Birmingham CL, Su J, Hlynsky JA, Goldberg EM. GAO M. The mortality rate from anorexia nervosa. Int J Eat Disord. 2005;38 2:143-6. doi:10.1002/eat.20164.

6. Isner JM, Roberts WC, Heymsfield SB, Yager J. Anorexia nervosa and sudden death. Ann Intern Med. 1985;102(1):49-52.

7. National Collaborating Centre for Mental Health. Core interventions in the treatment and management of anorexia Nervosa bulimia Nervosa and related eating disorders. London: National Institute for a Clinical Excellence; 2004.

8. American Psychiatric Association. Treatment of patients with eating disorders. America Journal of Psychiatry. 2006;163(7):4-54.

9. Whitelaw M, Gilbertson H, Lam PY, Sawyer SM. Does aggressive refeeding in hospitalized adolescents with anorexia nervosa result in increased hypophosphatemia? J Adolesc Health. 2010;46:577-82.

10. Rocks T, Pelly F, Wilkinson P. Nutritional management of anorexia nervosa in children and adolescent inpatients: The current practice of Australian dietitians. Nutrition Dietetics. 2014;71:100-7.

11. Earley T. Improving safety with nasogastric tubes: a whole-system approach. Nursing Times. 2019;115(12):50-1.

12. Best C. How to set up and administer an enteral feed via a nasogastric tube. Nurs Stand. 2017;31(45):42-7.

13. Ichimaru S. Methods of Enteral Nutrition Administration in Critically III Patients: Continuous, Cyclic, Intermittent, and Bolus Feeding. Nutr Clin Pract. 2018;33(6):790-5.

14. British Dietetic Association. Naso-Gastric Tube Feeding under restraint best practice guidelines for Dietitians July 2019.

15. NICE. Nutrition support in adults | Quality standards | NICE. 2019. https://www.nice.org.uk/guidance/qs24. June 282019.

16. Royal Collage of Psychiatry. CR168s Summary of Junior Marsipan: Management of really sick patients under 18 with Anorexia Nervosa. 2015.

17. Garber AK, Sawyer SM, Golden NH, et al. A systematic review of approaches to refeeding in patients with anorexia nervosa. Int J Eat Disord. 2016;49:293310.

18. Maginot TR, Kumar MM, Shiels J, Kaya W, Rhee KE. Outcomes of an inpatient refeeding protocol in youth with anorexia nervosa: Rady Children's Hospital San Diego/University of California, San Diego. Journal of eating disorders. 2017;5(1):1-10.

19. Skipper. Refeeding syndrome or refeeding hypophosphatemia: a systematic review of cases. Nutr Clin Pract. 2012;27(1):34-40.

20. Paccagnella A, Mauri A, Baruffi C, Berto R, Zago R, Mar, et al. Application criteria of enteral nutrition in patients with anorexia nervosa: correlation between clinical and psychological data in a "lifesaving" treatment. JPEN J Parenter Enteral Nutr. 2006;30(3):231-9.

21. Silber TJ, Robb AS, Orrell-Valente JK, Ellis N, Valadez-Meltzer A, Dadson MJ. Nocturnal nasogastric refeeding for hospitalized adolescent boys with anorexia nervosa. J Dev Behav Pediatr. 2004;25(6):415-8.

22. Madden S, Miskovic-Wheatley J, Wallis, et al. A randomized controlled trial of in-patient treatment for anorexia nervosa in medically unstable adolescents. Psychol Med. 2015;45:415-27.

23. Agostino H, Erdstein J, Di Meglio G. Shifting paradigms: continuous nasogastric feeding with high caloric intakes in anorexia nervosa. J Adolesc Health. 2013;53:590-4.

24. Parker E, Faruquie S, Anderson G, et al.Higher Caloric Refeeding Is Safe in Hospitalised Adolescent Patients with Restrictive Eating Disorders. Journal of Nutrition and Metabolism 2016:1-9.

25. Madden S, Mskovic-Whaetley J, Clarke S, Touyz S, Hay P, Kohn MR. Outcomes of a rapid refeeding protocol in Adolescent Anorexia Nervosa. Journal of Eating Disorders. 2015;3:8.

26. Kezelman S, Crosby RD, Rhodes P, Hunt C, Anderson G, Clarke S, Touyz S. Anorexia Nervosa, Anxiety, and the Clinical Implications of Rapid Refeeding. Front Psychol. 2018;9:P1097.

27. Fuller S, Street O, Hudson L, Nicholls D. Enteral feeding young people with anorexia nervosa under restraint in inpatient settings. British Journal of Mental Health Nursing. 2019;8:124-8.

28. Street K, Costelloe S, Wooton M, Upton S, Brough J. Structured, supported feeding admissions for restrictive eating disorders on paediatric wards. Arch Dis Child. 2016;101:836-8.

29. Couturier J, Mahmood A. Meal support therapy reduces the use of nasogastric feeding for adolescents hospitalized with anorexia nervosa. Eat Disord. 2009;17:327-32.

30. Falcoski P, Philpot U, Tan J, Hudson LD, Fuller SJ. Nasogastric tube feeding in line with new dietetic guidelines for the treatment of anorexia nervosa in a specialist children and adolescent inpatient unit: a case series. Journal of Human Nutrition and Dietetics 2020; May.

31. O'Connor G, Nicholls D, Hudson L, Singhal A. Refeeding Low Weight Hospitalized Adolescents With Anorexia Nervosa: A Multicenter Randomized Controlled Trial. 2016 Nutr Clin Pract. 2016;31:681-9.

32. Rizo S, Douglas JW, Lawrence JC. Enteral Nutrition via Nasogastric Tube for Refeeding Patients with Anorexia Nervosa: A Systematic Review. Nutr Clin Pract. 2019;34(3):359-70.

33. Hale D, Logomarsino JV. The use of enteral nutrition in the treatment of eating disorders: a systematic review. Eat Weight Disord. 2019;24(2):179-98. 
34. Myers E, McCrory D, Mills A, et al. Effectiveness of Assisted Reproductive Technology. Evidence Report/Technology Assessment No. 167 (Prepared by the Duke University Evidence-based Practice Center under Contract No. 290-02-0025.) AHRQ Publication No. 08-E012. Rockville: Agency for Healthcare Research and Quality; May; 2008.

35. Akgul S, Akdemir DP, Kara M, Derman, O'Cetin FC, Kabbur N. The understanding of risk factors for eating disorders in male adolescents. Int J Adolesc Med Health. 2016;28:97-105.

36. Akgul S, Pehlivanturk-Kizilkan M, Ors S, Derman O, Duzceker Y, Kanpur N. Type of setting for the inpatient adolescent with an eating disorder: Are specialized inpatient clinics a must or will the pediatric ward do? Turk J Pediatr. 2016;58:641-9.

37. Nehring I, Kewitz K, Von Kries R, Thyen U. Long-term effects of enteral feeding on growth and mental health in adolescents with anorexia nervosa-results of a retrospective German cohort study. Eur J Clin Nutr. 2014;68:171-7.

38. Neiderman M, Zarody M, Tattersall M, Lask B. Enteric feeding in severe adolescent anorexia nervosa: A report of four cases. Int J Eat Disord. 2000;28:470-5.

39. Robb AS, Silber TJ, Orwell-Valente JK, et al. 2002. Supplemental nocturnal nasogastric refeeding for better short-term outcome in hospitalized adolescent girls with anorexia nervosa. Am J Psychiatry 2002;159:1347-53.

40. Neiderman M, Farley A, Richardson J, Lask B. Nasogastric feeding in children and adolescents with eating disorders: toward good practice. Int J Eat Disord. 2001;29:441-8.

41. Gusella JL, Campbell AG, Lalji K. A shift to placing parents in charge: Does it improve weight gain in youth with anorexia? Paediatr Child Health. 2017;22:269-72.

42. Madden S, Morris A, Zurynski YA, Kohn M, Elliot EJ. Burden of eating disorders in 5-13-year-old children in Australia. Med J Aust. 2009;190:410-4.

43. Van Noort BM, Lohmar SK, Pfeiffer E, Lehmkul U, Winter SM, Kappel V. Clinical characteristics of early onset anorexia nervosa. Eur Eat Disord Rev. 2018;26:519-25.

44. Strik Lievers L, Curt F, Wallier J, et al. Predictive factors of length of inpatient treatment in anorexia nervosa. Eur Child Adolesc Psychiatry. 2009;18:75-84.

45. Halse C, Broughtwood D, Clarke S, Honey A, Kohn M, Madden S. Illumating multiple perspectives: meaning of nasogastric feeding in anorexia Nervosa. Europeon Eating Disorders Review. 2005;13:264-72.

46. Clausen L, Larsen JT, Bulik CM, Peterson L. A Danish register-based study on involuntary treatment in anorexia nervosa. Int J Eat Disord. 2018;51:121322.

47. Bayes A, Madden S. Early onset eating disorders in male adolescents: a series of 10 inpatients. AustralasPsychiatry. 2011;19:526-30.

48. Kodua M, MacKenzie JM, Smyth N. Nursing assistants' experiences of administering manual restraint for compulsory nasogastric feeding of young persons with anorexia nervosa. International Journal of Mental Health Nursing, 2020; June.

49. Rizzo SM, Douglas JW, Lawrence JC. Enteral Nutrition via Nasogastric Tube for Refeeding Patients With Anorexia Nervosa: A Systematic Review. Nutr Clin Prac. 2019;34(3):359-70.

\section{Figures}




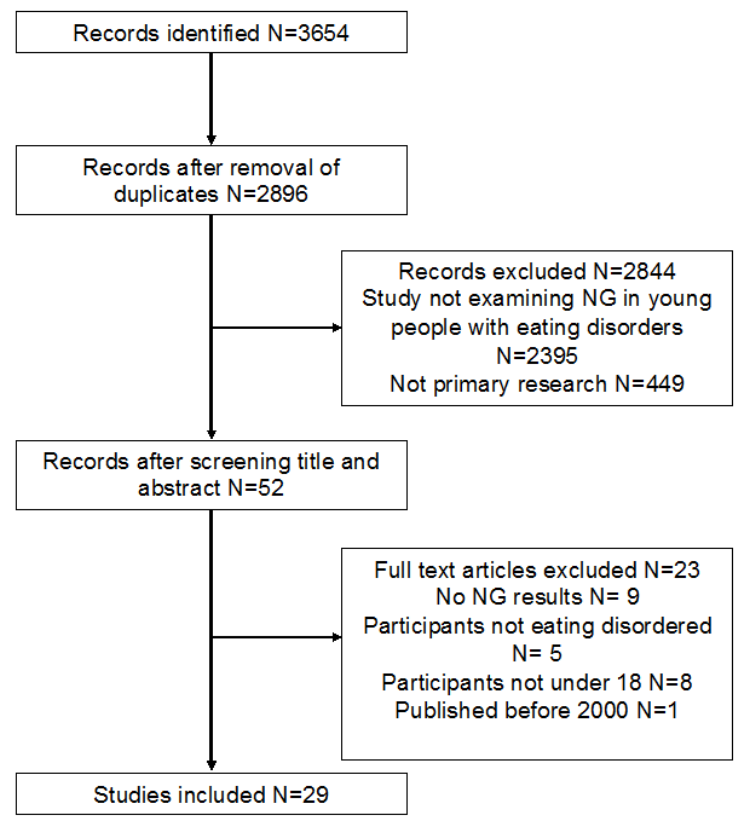

\section{Figure 1}

displaying PRISMA flowchart of methodology utilised to search databases for this systematic review of enteral feeding in young people with restrictive eating disorders. 